\title{
Commentaries
}

\section{Colonoscopic polypectomy and the incidence of colorectal cancer}

It has long been believed that colorectal cancer evolves from a precursor lesion, the adenomatous polyp. This concept was based on the elegant pathology studies from the St Mark's Hospital, London, published by LockhartMummery and Dukes in $1928,{ }^{1}$ and culminating in the concept of the polyp-cancer sequence published in 1975 by Muto and colleagues. ${ }^{2}$ The introduction of colonoscopy in the early 1970s, followed by the demonstration of the feasibility of colonoscopic polypectomy, provided the technology for the application of this concept to clinical practice. ${ }^{3}$ The entire colon could be examined, polyps identified and removed and, it was believed, colorectal cancer prevented. Evidence for this belief was provided by observations from the prospective National Polyp Study (NPS) in the USA that demonstrated a reduction in the incidence of colorectal cancer by $76-90 \%$ following colonoscopic polypectomy. ${ }^{4}$

The paper in this issue of Gut by Citarda et al addressed a common question - that is, can efficacy observed in a clinical trial be translated to effectiveness in clinical practice (see page 812 ). ${ }^{5}$ The study was a retrospective analysis of the incidence of colorectal cancer following colonoscopic polypectomy performed at medical centres throughout Italy, with many endoscopists participating in routine clinical practice. The study provides further evidence of the robustness of the NPS observations and adds validity to the clinical practice of colonoscopic polypectomy for the prevention of colorectal cancer. The belief initially formulated in the St Mark's studies has now been translated by evidence into clinical practice.

Citarda et al add further fuel to the increasing sentiment that colonoscopy may be the best screening option for the prevention of colorectal cancer. Guidelines published recently have suggested faecal occult blood testing every year and flexible sigmoidoscopy every five years as one option; or screening colonoscopy every 10 years; or double contrast barium enema every $5-10$ years along with flexible sigmoidoscopy. ${ }^{6}$ The radiological option has become less desirable considering the overall reduced interest in utilisation of the barium enema and the most recent observations of the NPS demonstrating that the double contrast barium enema, even when performed by experienced radiologists, has only a $50 \%$ sensitivity for the detection of adenomatous polyps, even those $1 \mathrm{~cm}$ in size or larger. ${ }^{7}$ Recent reports from the USA have demonstrated that screening colonoscopy is feasible, with a high completion rate to the caecum, a very low complication rate, and a manageable outcome of approximately $10 \%$ neoplasia with advanced pathology, including significant adenomas and cancer. $^{89}$ This rate of advanced pathology provides excellent stratification, separating those $(90 \%)$ who may not need any further follow up from those that need further colonoscopy follow up. This gives us some indication of the resulting burden on the available medical resources. These observations, as well as the present study in Gut, strengthen the case for screening colonoscopy as a desirable option for asymptomatic general population screening.
Do we need additional evidence to support screening colonoscopy which is rapidly becoming the preferred option based on available evidence ${ }^{10}$ The evidence we now have is indirect and addresses feasibility. The studies of Lieberman and colleagues ${ }^{8}$ and Imperiale and colleagues ${ }^{9}$ of feasibility report neoplastic findings and performance of colonoscopy. The NPS and Citarda et al studies provide an estimate of the reduction in the incidence of colorectal cancer following colonoscopic polypectomy. However, these studies were in adenoma bearing cohorts as observational studies could overestimate the magnitude of the effect in the general population. A randomised controlled trial of screening colonoscopy in the general population could answer the following questions: the magnitude of the reduction in incidence and mortality of colorectal cancer in the general population as a result of screening colonoscopy; complication rate of widespread screening colonoscopy; duration of the benefit; harms versus benefits; cost effectiveness; acceptance of screening colonoscopy in the general population; and comparison of efficacy of screening colonoscopy with that observed in randomised controlled trials of screening flexible sigmoidoscopy ${ }^{11} 12$ and faecal occult blood testing. ${ }^{6}$ The age at which screening colonoscopy is to be offered is an important consideration. If we adhere to present guidelines of screening colonoscopy every 10 years beginning at age 50 , resources could be enormously overwhelmed. A properly timed "once in a lifetime" screening colonoscopy could provide an enormous impact while keeping the added burden on medical resources at a lower level.

Why do we need a long term randomised trial of screening colonoscopy in the general population? Universal acceptance of screening colonoscopy by the public, medical community, and providers requires strong direct evidence of effectiveness, data on benefit versus harms, data on cost effectiveness and intervals between screening examinations, and follow up surveillance examinations in those with neoplastic findings. We need to have a full understanding of all aspects of this strategy to implement it optimally in the millions of people at risk.

Issues on the effectiveness of screening colonoscopy will arise when strong evidence of screening flexible sigmoidoscopy is reported over the next few years and added to the strong evidence of faecal occult blood testing. Questions will be asked regarding the "projected" higher effectiveness of screening colonoscopy, and at what cost to the patient and to society in terms of harms, resources, and finances. We believe that screening colonoscopy is the preferred option for colorectal cancer screening but we must test out this belief. We reiterate the statement written in our guidelines paper published in 1997. "Because this strategy (screening colonoscopy) is potentially so effective, there is an urgent need for better evidence of its effects in prospective control trials." "This belief needs strong evidence to be translated into effective universal implementation.

S J WINAWER

Gastroenterology and Nutrition Service,

Department of Medicine,

Memorial Sloan-Kettering Cancer Center, 1275 York Avenue,

New York 1002, USA

Department of Epidemology and Biostatistics,

Memorial Sloan-Kettering Cancer Center,

New York, USA 
Correspondence to: SJ Winawer. winawers@mskcc.org

1 Lockhart-Mummery H, Dukes C. The pre-cancerous changes in the rectum and colon. Surg Gynecol Obst 1928;46 (No 5).

and colon. Surg Gynecol Obst 1928; The evolution of cancer of the colon and rectum. Cancer 1975;36:2251-70.

3 Wolf WI, Shinya H. Polypectomy via the fiberoptic colonoscope: removal of neoplasms beyond the reach of the sigmoidoscope. $N$ Engl $f$ Med 1973;288:329-32.

4 Winawer SJ, Zauber AG, Ho MN, et al. Prevention of colorectal cancer by colonoscopic polypectomy. N Engl f Med 1993;329:1977-81.

5 Citarda F, Tomaselli G, Capocaccia R, et al. Efficacy in standard clinical practice of colonoscopic polypectomy in reducing colorectal cancer incidence. Gut 2001;48:812-5.

6 Winawer SJ, Fletcher RH, Miller L, et al. Colorectal cancer screening: clinical guidelines and rationale. Gastroenterology 1977;112:594-642.
7 Winawer SJ, Stewart ET, Zauber AG, et al. Colonoscopy and double contrast barium enema for surveillance following polypectomy. $N$ Engl f Med 2000;342:1766-72.

8 Lieberman DA, Weiss DG, Bond JH, et al. Use of colonoscopy to screen asymptomatic adults for colorectal cancer. N Engl f Med 2000;343:162-8.

9 Imperiale TF, Wagner DR, Lin CY, et al. Risk of advanced proximal neoplasms in asymptomatic adults according to the distal colorectal findings. N Engl f Med 2000:343:169-74.

10 Rex DK, Johnson DA, Lieberman DA, et al. Colorectal cancer prevention 2000: screening recommendations of the American College of Gastroenterology. Am f Gastroenterol 2000; 95:868-77.

11 Gohagan JK, Prorok PC, Kramer BS, et al. The prostate, lung, colorectal and ovarian screening trial of the National Cancer Institute. Cancer 1995; 75:1869-73

12 Atkin WS, Hart A, Edwards R, et al. Uptake, yield of neoplasia, and adverse effects of flexible sigmoidoscopy screening. Gut 1998:42:560-5.

\section{Small fry starting to make waves}

The liver, perhaps because of its crucial role in metabolism and detoxification of many potentially hazardous xenobiotics, has evolved not one but three stem cell populations. The one that usually operates is the hepatocyte population itself. Hepatocytes were formerly (and incorrectly) considered to have a limited division potential because only 2-3 rounds of cell division occurred after a two thirds partial hepatectomy but this was all that was required to restore liver mass! However, their full division potential has been unmasked through the study of models of hepatocyte transplantation, and they fully deserve the appellation of "functional stem cells" with at least some of them being capable of in excess of 100 divisions. A second population comes into play when either hepatocyte regeneration is compromised after injury or when parenchymal damage is particularly severe. If we did not already know it, rats and humans are different and this extends to the intrahepatic biliary tree. In rats, the terminal bile ductules (canals of Hering) barely extend beyond the portal tract, and the so-called ductular oval cell response that occurs after parenchymal injury when hepatocyte regeneration is inhibited emanates from the intraportal bile ducts and canals of Hering. ${ }^{1}$ On the other hand, in humans, the canals of Hering extend into the proximate third of the hepatic lobule, and the ductular reactions that are seen after massive necrosis are considered to be the proliferative reactions of these extended canals of Hering, ${ }^{2}$ being akin to a trip wire that "senses" severe injury. Thus in both rats and humans, the cholangiocyte population is considered to harbour a facultative stem cell population that acts as a "back up" to the functional stem cells. More recently, a third liver stem cell candidate has become apparent, namely bone marrow stem cells that appear to be able to differentiate into hepatocytes and cholangiocytes, ${ }^{34}$ and moreover are capable of making a substantial contribution to liver repopulation in a murine model of metabolic liver disease. ${ }^{5}$

The now classical experiments on epidermal carcinogenesis indicated that the target cell for genotoxic chemicals is likely to be a long lived cell (that is, a stem cell) and indeed popular opinion opines that neoplasia, like metaplasia, is a stem cell disease. So what is the carcinogen target cell for the development of primary tumours of the liver? As itemised above, there are at least three possible different cellular targets, ${ }^{6}$ although for tumours of the biliary epithelia it is likely to be directly from a cholangiocyte, but that cell could have been bone marrow derived! In 1998 in the UK, primary tumours of the liver accounted for only $<1 \%$ of the total cancer burden and for $1 \%$ of deaths from cancer (www.crc.org.uk). However, in this issue of Gut, Simon Taylor-Robinson and colleagues flag up a seemingly inexorable rise in the number of deaths in England and Wales from intrahepatic cholangiocarcinoma, ${ }^{8}$ a relatively rare cancer with a dismal prognosis (see page 816). They report a 15 -fold increase in age specific mortality rates in the 45 and over age groups over a 30 year period, resulting in this malignancy now being the commonest primary liver tumour and responsible for more deaths than hepatocellular carcinoma in England and Wales. In China, Japan, and South East Asia, infection by liver flukes such as Clonorchis sinensis and Opisthorchis viverrini is endemic, and here the chronic biliary irritation associated with these food borne trematodes is a predisposing factor: in areas of Thailand, with the highest incidence of $O$ viverrini infection, the incidence of cholangiocarcinoma is 40 times the highest incidence outside Thailand and cases of cholangiocarcinoma outnumber cases of hepatocellular carcinoma.

So what is the aetiology of intrahepatic cholangiocarcinoma in England and Wales, and is increased proliferation of the putative target cell (cholangiocyte) a significant risk factor? Primary sclerosing cholangitis, an inflammatory disease of bile ducts, is certainly a predisposing factor in a minority of cases, and in models of chemically induced hepatocellular carcinoma many established hepatocarcinogens are only effective if coupled to enhanced hepatocyte turnover in an otherwise essentially mitotically quiescent organ. An eminent hepatopathologist Ken Weinbren once remarked that "bile ducts proliferate at the drop of a hat" and apart from their function as facultative stem cells, cholangiocyte proliferation can be stimulated by the likes of bile acids and oestrogens, ${ }^{9}$ and indeed many compounds with oestrogenic properties can probably enter the food chain. As to the identity of the carcinogenic agent(s), we are largely in the dark; polychlorinated biphenyls are ubiquitous and persistent environmental contaminants whose soil concentrations have been rising for some time, even before the rises in intrahepatic cholangiocarcinoma reported here. Rated as "probable human carcinogens" by the IARC, they may be linked to breast cancer but an association with liver tumours has yet to be proved. The genetic alterations implicated in the development and progression of intrahepatic cholangiocarcinoma are often the usual culprits such as TP53, mdm2, K-ras, and INK4a, although a dysfunctional p53 pathway through TP53 mutation and/or $\mathrm{Mdm} 2$ overexpression seems to be the most consistent reported abnormality. ${ }^{10}$ Interestingly, the types of mutations in the TP53 gene in European patients are dissimilar from those in East Asian patients suggesting different environmental factors are at work. ${ }^{11}$ 
Although there are widely acknowledged increases in the levels of malignant melanoma and prostate cancer, for example, much bigger hitters in the league of cancer statistics, the report by Taylor-Robinson et al seems to highlight a much more striking increase in a tumour still relatively uncommon in the UK. This increase appears "real" and could not reasonably be attributed to "diagnosis transfer" or the widespread adoption of improved investigative procedures. Clearly the search for carcinogenic agents in bile should be a high priority.

Department of Histopathology, ICSM at Hammersmith Campus, Du Cane Road, London W12 ONN, UK

m.alison@ic.ac.uk

1 Alison M, Golding M, Lalani EN, et al. Wholesale hepatocytic differentiation in the rat from ductular oval cells, the progeny of biliary stem cells. $\mathcal{F}$ Hepatol 1997;26:343-52.
2 Theise ND, Saxena R, Portmann BC, et al. The canals of Hering and hepatic stem cells in humans. Hepatology 1999;30:1425-33.

3 Alison MR, Poulsom R, Jeffery R, et al. Hepatocytes from non-hepatic adult stem cells. Nature 2000;406:257.

4 Theise ND, Nimmakayalu M, Gardner R, et al. Liver from bone marrow in humans. Hepatology 2000;32:11-16.

5 Lagasse E, Connors H, Al-Dhalimy M, et al. Purified hematopoietic stem cells can differentiate into hepatocytes in vivo. Nat Med 2000;6:1229-34.

6 Sell S. Heterogeneity and plasticity of hepatocyte lineage cells. Hepatology 2001;33:738-50

7 Crosby HA, Kelly DA, Strain AJ. Human hepatic stem-like cells isolated using c-kit or CD34 can differentiate into biliary epithelium. Gastroenterology 2001;120:534-44.

8 Taylor-Robinson SD, Toledano MB, Artora S, et al. Increase in mortality rates from intrahepatic cholangiocarcinoma in England and Wales. Gut $2001 ; 48: 816-20$

9 Alvaro D, Alpini G, Onori P, et al. Estrogens stimulate proliferation of intrahepatic biliary epithelium in rats. Gastroenterology 2000;119:1681-91.

10 Della Torre G, Pasquini G, Pilotti S, et al. TP53 mutations and mdm2 protein overexpression in cholangiocarcinomas. Diagn Mol Pathol 2000; 9:416.

11 Tullo A, D'Erchia AM, Honda K, et al. New p53 mutations in hilar cholangiocarcinoma. Eur f Clin Invest 2000;30:798-803.

\section{Iron overload in Montpelier}

Since the original discovery of the haemochromatosis gene (HFE) in $1996,{ }^{1}$ there have been two genetic mutations that have dominated clinical studies. The C282Y mutation $(845 \mathrm{G} \rightarrow \mathrm{A})$ has attracted the most attention as the majority of typical haemochromatosis patients are homozygotes for this mutation. This has increasingly led to the use of genetic testing for the diagnosis of haemochromatosis and in many cases has alleviated the need for liver biopsy. In comparison with most other genetic diseases, it remains a marvel that a single mutation explains most clinical cases. Furthermore, in vitro studies have demonstrated that the C282Y mutation disrupts the normal presentation of the HFE protein on the cell surface. ${ }^{2}$ The H63D mutation $(187 \mathrm{G} \rightarrow \mathrm{C})$ was described in the original description of the $H F E$ gene but has been relegated to a minor clinical role in comparison with the $\mathrm{C} 282 \mathrm{Y}$ mutation. It is more prevalent than the C282Y mutation with approximately one in five of the European population carrying the mutation. Despite this high prevalence, it is not well represented in descriptions of large series of haemochromatosis patients defined on the basis of iron overload. The H63D mutation has also not been shown to cause the same intracellular processing defect as the $\mathrm{C} 282 \mathrm{Y}$ mutation.

In this issue of Gut, Aguilar-Martinez and colleagues describe a series of 56 H63D homozygotes with varying degrees of iron abnormalities (see page 836). ${ }^{3}$ Although this is not the first description of iron overload in H63D homozygotes, it represents a large series in a referred sample of 968 cases. The authors have tried to eliminate known risk factors such as alcoholism and viral hepatitis and searched for other genetic mutations associated with iron overload. Fifty of their cases had iron abnormalities which ranged from slight elevations in transferrin saturation to six cases of cirrhosis and two with hepatocellular carcinoma. The presence of liver dysfunction is an unexpected observation as in compound heterozygotes $(\mathrm{C} 282 \mathrm{Y} / \mathrm{H} 63 \mathrm{D})$ liver damage is uncommon in the absence of another risk factor. The authors acknowledge the effects of referral or ascertainment bias in their study and suggest that population based studies are required to establish the clinical significance of the H63D homozygote. In fact, many of these population studies have been done and many more are in progress. In the screening of 10198 patients at a health appraisal clinic in San Diego, there were 203 H63D homozygotes. Mean serum ferritin in these homozygotes was $71 \mu \mathrm{g} / 1$ in women and $133 \mu \mathrm{g} / 1$ in men. ${ }^{4}$ Population studies in Australia ${ }^{5}$ and Canada $^{6}$ in which over 8222 people were screened did not detect any H63D homozygotes in cases of iron overload.

Why are there so many iron loaded H63D homozygotes in Montpelier, France, that have not been described in other countries? If a referral bias was the sole explanation, it would be expected that other iron overload referral centres would have a large number of cases. At our own clinic, we have evaluated 25 referred H63D homozygotes with a mean serum ferritin of $760 \mu \mathrm{g} / 1$ (range $7-8142$ ). The study of siblings of these H63D proband cases would be another approach to reduce referral bias effects. This approach has recently been reported in C282Y homozygous siblings. ${ }^{7}$ Is it possible that the H63D mutation in these patients is on a haplotype containing another iron loading gene? Different haplotypes with the H63D mutation have been described in Sri Lanka. ${ }^{8}$ If a regional variation was the explanation, we would expect to see many iron loaded H63D cases in Spain and in screening studies in progress in Rennes, France. The authors point out that H63D homozygotes are very common in their community (3\%) and also that $3 \%$ of their iron loaded cases carried no HFE mutations, and therefore some of the cases may incidentally be H63D homozygotes without a direct causal relationship. In clinical practice, it seems reasonable to test for both the C282Y and H63D mutations in iron loaded patients. Advances in diagnostic techniques have allowed for the rapid detection of both HFE mutations. The use of H63D testing in population screening is less certain as it seems likely that most H63D homozygotes will not have iron overload, and so they should not be subjected to the potential risks of stigmatisation, labelling, and genetic discrimination. It continues to be important in all haemochromatosis genotyping studies to include control patients as attribution of symptoms to genotype or iron parameters can be difficult. This current study is provocative because it presents a large number of cases of iron overload in H63D homozygotes. It may stimulate the discovery of other genetic or environmental factors contributing to iron overload in these 
patients. The large population screening studies which are in progress in many countries should resolve the important issues surrounding the clinical relevance of the H63D homozygote.

P C ADAMS

Department of Medicine, London Health Sciences Centre, 339 Windermere Road, London Ontario, Canada padams@uwo.ca

1 Feder JN, Gnirke A, Thomas W, et al. A novel MHC class I-like gene is mutated in patients with hereditary haemochromatosis. Nat Genet mutated in patien.

2 Feder J, Tsuchihashi Z, Irrinki A, et al. The haemochromatosis founder mutation in HLA-H disrupts beta-2-microglobulin interaction and cell surface expression. f Biol Chem 1997;272:14025-8.
3 Aguilar-Martinez P, Bismuth M, Picot MC, et al. Variable phenotypic presentation of iron overload in H63D homozygotes: are gene modifiers the cause? Gut 2001;48:836-42.

4 Beutler E, Felitti V, Gelbart T, et al. The effect of HFE genotypes on measurement of iron overload in patients attending a health appraisal clinic. Ann Intern Med 2000;133:329-37.

5 Olynyk J, Cullen D, Aquilia S, et al. A population-based study of the clinical expression of the haemochromatosis gene. N Engl f Med 1999;341:718-24.

6 Adams PC, Kertesz AE, McLaren C, et al. Population screening for haemochromatosis: a comparison of unbound iron binding capacity, transferrin saturation and $\mathrm{C} 282 \mathrm{Y}$ genotyping in 5,211 voluntary blood donors. Hepatology 2000;31:1160-4

7 Bulaj Z, Ajioka RS, Phillips J, et al. Disease-related conditions in relatives of patients with haemochromatosis. N Engl f Med 2000;343:1529-35.

8 Rochette J, Pointon JJ, Fisher CA, et al. Multicentric origin of haemochromatosis gene (HFE) mutations. Am f Hum Genet 1999;64:1056-62. 